OnLine Journal of Biological Sciences 8 (4): 73-79, 2008

ISSN $1608-4217$

(C) 2008 Science Publications

\title{
Development of Three Bacteria Consortium for the Bioremediation of Crude Petroleum-oil in Contaminated Water
}

\author{
Abdualdaim Mohammed Mukred, Aidil Abd Hamid, Ainon Hamzah and \\ Wan Mohtar Wan Yusoff \\ School of Biosciences and Biotechnology, Faculty of Science and Technology, \\ Universiti Kebangsaan Malaysia, 43600 Bangi, Selangor, Malaysia
}

\begin{abstract}
We have to developed active microbial consortium that could be of higher degradation of crude oil contaminated groundwater, wastewater aeration pond and biopond at the oil refinery Terengganu Malaysia. Among four isolates that showed good growth only three different isolates (Acinetobacter faecalis WD2, Staphylococcus. sp DD3 and Neisseria elongate TDA4.) were selected based on the growth ability and degradation. Significant growth and effectiveness of hydrocarbon biodegradation of the bacterial consortium examined bacterial strains and their mixtures in both were observed after 5, 10 and 15 days of degradation. Gas chromatography showed that more than 96 and $98 \%$ degradation of total hydrocarbon by consortia sp respectively.
\end{abstract}

Key words: Biodegradation, crude oil contaminated water

\section{INTRODUCTION}

Chemical treatment includes direct injection of chemical oxidants into contaminated soil and groundwater thereby altering native aquatic chemistry and biology. Biological treatment most commonly involves the breakdown of contamination into nontoxic forms using microbiological processes ${ }^{[1]}$. Thus, bioremediation may be defined as the use of living organisms to remove environmental pollutants from soil, water and gases ${ }^{[2]}$.The advantages of employing mixed cultures as opposed to pure cultures in bioremediation have been widely demonstrated. It could be attributed to the effects of synergistic interactions among members of the association. The mechanisms in which petroleum degraders' benefit from synergistic interactions may be complex. It is possible that one species removes the toxic metabolites (that otherwise may hinder microbial activities) of the species preceding it. It is also possible that the second species are able to degrade compounds that the first are able to only partially ${ }^{[3]}$. Further research should be directed towards understanding the roles of individual members in influencing the effectiveness of a microbial consortium. Demonstrated a consortium of 8 strains made up of members of 6 genera to be able to effectively degrading crude oil ${ }^{[4]}$. Interestingly, only 5 of these strains were able to grow in pure cultures using a variety of hydrocarbons. However, when the other 3 strains were removed from the consortium, the effectiveness of the mixed culture was remarkably reduced. These further support the theory that each member in a microbial community has a significant role and may need to depend on the presence of other species or strains to be able to survive. They are produced by many bacterial strains that can degrade or transform the components of petroleum products. They are non-toxic, non-hazardous, biodegradable and environmentally friendly compounds ${ }^{[5]}$.

\section{MATERIALS AND METHODS}

Source of microorganisms, media and culture condition: Bacterial strains were isolated from samples collected from groundwater and wastewater aeration pond and biopond located at the Terengganu oil refinery, Malaysia. $10 \mathrm{ml}$ of each sample were washed with $90 \mathrm{~mL}$ saline and filtered with membrane. Incubation was carried out for $24 \mathrm{~h}$ at $37^{\circ} \mathrm{C}$ on nutrient agar plate. Mineral Salts Medium $(\mathrm{MSM})^{[6]}$ was prepared by dissolving $1.8 \mathrm{~g} \mathrm{~K}_{2} \mathrm{HPO}_{4}, 4.0 \mathrm{~g} \mathrm{NH} \mathrm{N}_{4} \mathrm{CI}, 0.2 \mathrm{~g}$ $\mathrm{MgSO}_{4} .7 \mathrm{H}_{2} \mathrm{O}, 0.1 \mathrm{~g} \mathrm{NaCL}, 0.01 \mathrm{~g} \mathrm{FeSO}_{4} .7 \mathrm{H} 2 \mathrm{O}$ in $1 \mathrm{~L}$ of distilled water. Bacteriological agar was added $(15 \mathrm{~g} / 1)$ to the solution where solid basal medium was required. The $\mathrm{pH}$ was adjusted to 6.90 and the medium was autoclaved at $121^{\circ} \mathrm{C}$ for $15 \mathrm{~min} .1 .0 \%(\mathrm{v} / \mathrm{v})$ tapis crude

Corresponding Author: Abdualdaim Mohammed Mukred, School of Biosciences and Biotechnology, Faculty of Science and Technology, Universiti Kebangsaan Malaysia, UKM, 43600 Bangi, Selangor, Malaysia 
OnLine J. Biol. Sci., 8 (4): 73-79, 2008

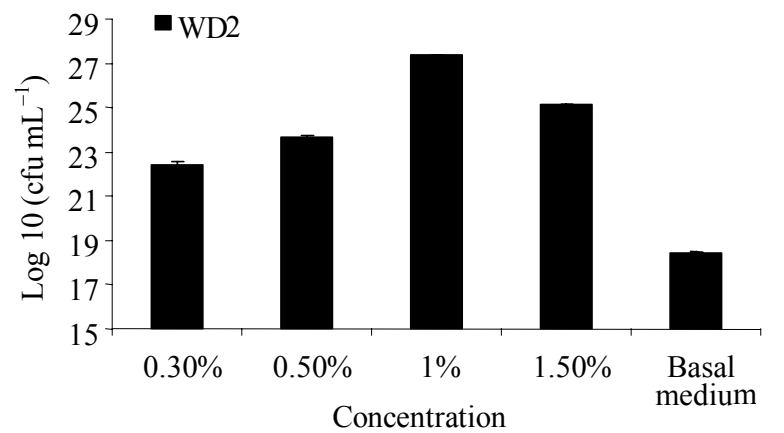

(a)

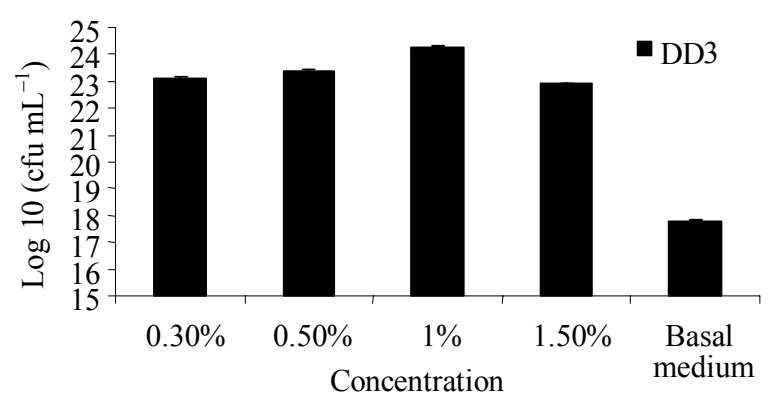

(b)

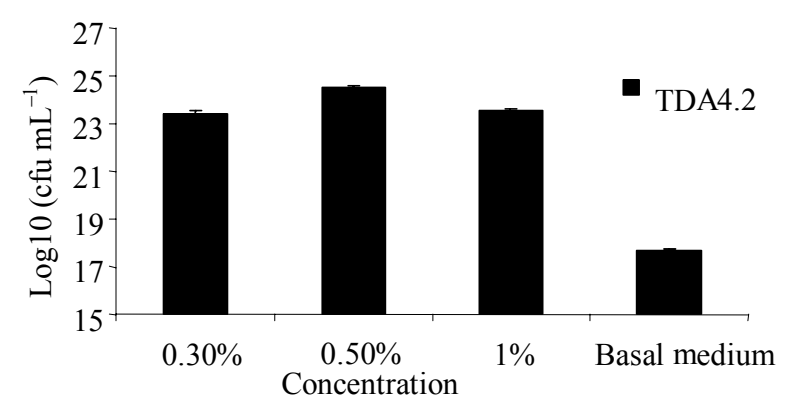

(c)

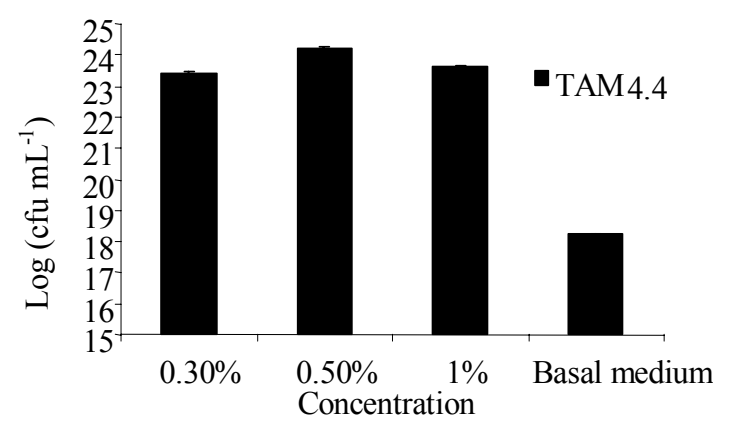

(d)

Fig. 1: Effect of different concentration of peptone on growth of isolates (a) Acinetobacter faecalis (b) Staphylococcus. sp (c) Neisseria elongate and (d), Pseudomonas putida


Fig.2: Growth of isolates (WD2, DD3, and TDA4.2) as consortium throughout 5,10 and 15 days in the MSM medium with $1 \%$ Tapis crude oil

oil was added as the sole carbon source and complex vitamin solution $\left(1.0 \mathrm{~mL}^{-1}\right)$ according to manufacturer's suggestion.

Effect of nitrogen source on growth and biodegradation: To determine the effect of the organic nitrogen source on biodegradation, the inorganic nitrogen sources of the basal medium were substituted by the addition of the following; peptone at 
concentrations of $0.30,0.50,1$ and $1.50 \%(\mathrm{v} / \mathrm{v})$. Cultivations were carried out after 5 days and the growth was measured as CFU count

Construction of bacterial consortia: The three different isolates that demonstrated good growth were chosen to construct consortia of hydrocarbon degraders. In total, three different bacteria were constructed consortia and tested for in this study to confirm their biodegradation capabilities.

Preparation of consortia inoculum: The isolates were grown separately in NB and processed to yield separate suspensions with an absorbance reading of 0.5 at 550 nM. Specific aliquots of the bacterial inoculum were then separately added into normal saline solution to give a final combined inoculum concentration of $10 \%$ $(\mathrm{v} / \mathrm{w})$.

Extraction of residual crude oil for Gas Chromatography (GC) analysis: Crude oil was extracted with a pre-cleaned separating funnel, following the modified methods of ${ }^{[7]} .50 \mathrm{~mL}$ of sample $(\mathrm{BM}+$ crude oil + bacteria), were then centrifuged at $4000 \mathrm{rpm}$ for $15 \mathrm{~min}$. The separating funnel was stoppered with a glass stopper and it was shaken vigorously. Vapour was carefully vented out through the stopcock. The process of venting and vigorous shaking was repeated for several minutes and the mixture was allowed to separate into two phases. After phases have been separated in the funnel, chloroform was collected in a $250 \mathrm{~mL}$ pre-cleaned bottle. The extraction was repeated with $25 \mathrm{~mL}$ chloroform twice. Then, it was filtered and dried by passing it through of $3 \mathrm{~g}$ pre-combusted $\mathrm{Na}_{2} \mathrm{SO}_{4}\left(150^{\circ} \mathrm{C}\right.$ for $3 \mathrm{~h}$ in incubation) and collected in a round bottom flask. The extract was then evaporated by using a rotary evaporator. The crude oil extracted was washed with $2 \mathrm{~mL}$ chloroform. The solvents were removed under a gentle stream (dried in fume chamber) for 7 days. The residue was reconstituted with $1 \mathrm{~mL}$ of chloroform and analyzed by GC.

Analyses of extract: A detailed analysis of the hydrocarbon extract was performed by Gas Chromatography GC. The clarus GC 500 was equipped with a split injector (split ratio 50/1) and a Flame Ionization Detector FID both set at $300^{\circ} \mathrm{C}$; gas carrier

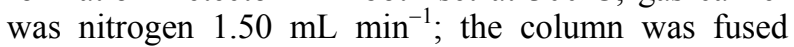
silica capillary column $(30.0 \mathrm{mx} 0.32 \mathrm{~mm}$, film thickness $0.25 \mu \mathrm{m}$ ); temperature programming was 60 $320^{\circ} \mathrm{C}, 5^{\circ} \mathrm{C} \mathrm{min}^{-1}$, injection volume $1 \mu 1$.
Table1: Three bacteria consortium selected for biodegradation

\begin{tabular}{ll}
\hline Bacteria & Consortium \\
Acinetobacter faecalis and Staphylococcus. sp & $\mathrm{A}+\mathrm{B}$ \\
Acinetobacter faecalis and Neisseria elongate & $\mathrm{A}+\mathrm{C}$ \\
Staphylococcus. sp and Neisseria elongate & $\mathrm{B}+\mathrm{C}$ \\
Acinetobacter faecalis, Staphylococcus. sp and & $\mathrm{A}+\mathrm{B}+\mathrm{C}$ \\
Neisseria elongate & \\
\hline
\end{tabular}

\section{RESULTS AND DISCUSSIONS}

Effect of peptone on growth and degradation: The bacterial growths in different percentages of peptone as nitrogen source were studied (Fig. 1). All four isolates showed good growth at different peptone concentrations $0.3,0.5,1.0$ and $1.50 \%$. Optimum growths were observed at percentages 1\% Tapis crude oil on the 5 day of incubation. Among the four isolates, isolate WD2 and DD3 showed a maximum growth with $1 \%$ peptone while, isolates TDA4.2 and TAM 4.4 showed a maximum growth with $0.5 \%$ peptone. From this study, $1 \%$ peptone was the best nitrogen source tested for growth compared to the basal medium. From four selected isolates, only three isolate showed good selected for consortium (Table 1). However, increasing the concentration of peptone from $1-1.50 \%$ significantly decreases growth and degradation. This is in agreement with the finding by ${ }^{[8]}$ that due to enhanced growth; degradability of oil was better in a medium containing $1 \%$ peptone, compared to medium containing either ammonium sulfate or potassium nitrate. Also reported cell growth increase in the medium with $1 \%$ peptone for pseudomonas fluoresences $\mathrm{FS}^{[9]}$.

Evaluation of bacterial consortia: Growth and degradation of $1 \%$ tapis crude oil: The growth of three selected isolates as consortia that could effectively remove hydrocarbon pollutants, following 5, 10 and 15 days incubation at $37^{\circ} \mathrm{C}$, pH 7 and with agnation at 150 rpm was measured as CFU counts and degradation was measured using GC (Fig. 2). The consortia were analyzed for the extent of biodegradation in medium containing 1\% tapis crude oil contaminated water (Fig. 4 and 5).

Consortia A and B that consisted of the WD2 and DD3 showed increased growth in the cell numbers from $243 \times 10^{22}-178 \times 10^{23} \mathrm{CFU} \mathrm{mL}^{-1}$ within 5 and 10 day and the end of the 15 day deceased to $105 \times 10^{21} \mathrm{CFU} \mathrm{mL}^{-1}$ (Fig. 2).

The consortia showed $97.50 \%$ degraded found to degraded $100 \%$ short-chain and intermediate while was still limited to longer-chain aliphatic compounds 




Fig.3: Growth of consortium $\mathrm{ABC}$ and degradation at 5,10 , and 15 days in the MSM medium with $1 \%$ Tapis crude oil

between 97.56 and $97.52 \%$ of $\mathrm{C}_{23}-\mathrm{C}_{34}$ remained in the presence of longer-chain between 2.44 and $2.48 \%$ of $\mathrm{C}_{23}-\mathrm{C}_{34}$ remained in the present of longer-chain after 5 days (Fig. 4)

The second combination of the two isolates WD2 and TDA2.4 (Mixture A and C) when tested on 1\% Tapis crude oil at 5 days of incubation. The cell numbers of strain increased from $148 \times 10^{21}$ to $254 \times 10^{22}$ $\mathrm{CFUmL}^{-1}$ within 5 and 10 day at the end of the 15 day study, cell counts decreased to $89 \times 10^{21} \mathrm{CFUmL}^{-1}$ (Fig. 2). While reduction in amounts of hydrocarbons was detected during gas chromatographic analysis was degraded $96.88 \%$ that $100 \%$ short-chain and intermediate while was still limited to longer-chain aliphatic compounds between 97.99 and $91.33 \%$ of $\mathrm{C}_{22}$ to $\mathrm{C}_{35}$, while between 2.01 and $8.66 \%$ remained in the present of longer-chain after 5 days (Fig. 4)

The third consortia of the two isolates DD3 and TDA2.4 (Mixture B and C) when tested on 1\% Tapis crude oil at 5 days of incubation. The bacterial cell numbers of strain increased from $156 \times 10^{20}$ to $188 \times 10^{21}$ $\mathrm{CFUmL}^{-1}$ within 5 and 10 day at the end of the 15 day study, cell counts decreased to $75 \times 10^{20} \mathrm{CFUmL}^{-1}$ (Fig.2). The reduction in the amounts of hydrocarbons was detected during gas chromatographic analysis was degraded $96.77 \%$ that $100 \%$ short-chain and intermediate while was still limited to longer-chain aliphatic compounds between 96.90 and $96.17 \%$ of $\mathrm{C}_{26}$ to $\mathrm{C}_{37}$ after 5 days (Fig.4)

The fourfold consortia of three isolates WD2, DD3 and TDA2.4 (Mixture A, B and C), the cell of Consortium A, B and C increased from $135 \times 10^{25}$ to $175 \times 10^{26}$ a CFU mL $\mathrm{mL}^{-1}$ within 5 and 10 day at the end of the 15 day study, cell counts decreased to $134 \times 10^{25}$ CFU mL ${ }^{-1}$ (Fig.2).
The higher levels of detected hydrocarbon during gas chromatographic analysis removal were seen with consortium A, B and C 98.23 degraded after 5 days that $100 \%$ short-chain, intermediate while was still limited to longer-chain aliphatic compounds between 98.13 and $95.58 \%$ of $\mathrm{C} 27$ to $\mathrm{C} 36$ (Fig.4). further the consortium $\mathrm{A}, \mathrm{B}$ and $\mathrm{C}$ was efficient higher levels of hydrocarbon at degraded $98.25 \%$ after 10 days that $100 \%$ short-chain, intermediate while was still limited to longer-chain aliphatic compounds between 98.40 and $97.21 \%$ of $\mathrm{C}_{28}$ to $\mathrm{C}_{34}$ after 10days (Fig. 5). While none aliphatic compound degradation was seen higher after 15 day $98.50 \%$ degraded between 98.35 and $97.12 \%$ of $\mathrm{C}_{28}$ to $\mathrm{C}_{33}$ compared to 10day (Fig. 3). Consortium mixture A, $\mathrm{B}$ and $\mathrm{C}$ degraded $100 \%$ short-chain and intermediate while was limited to longer-chain aliphatic compounds compared to other consortia.

The bacterial consortium showed that it could degrade up to a maximum of $98 \%$ after 15 days incubation. Higher levels (up to100\%) of degraded hydrocarbon were seen in the short-chain and medium chain alkanes compared to the longer chain alkanes. However, after 15 days of incubation post contamination reduction was seen in all the investigated hydrocarbons although the reduction was more significant in the short-chain, medium-chain aliphatic compounds and aromatic compounds compared to longer-chain. This is in agreement since short-chain and medium-chain alkanse are generally easily degraded due to their lower hydrophobicity. Reported that the maximum degradation was achieved of treatment nalkanes in the range of $\mathrm{C}_{8}-\mathrm{C}_{11}$, which were degraded completely followed by $\mathrm{C}_{12}-\mathrm{C}_{21}, \mathrm{C}_{22}-\mathrm{C}_{31}$ and $\mathrm{C}_{32}-\mathrm{C}_{40}$ with degradations percentage of 100, 83-98, 80-85 and $57-73 \%$ respectively using bacterial consortium ${ }^{[10]}$. In another report by ${ }^{[11]}$, the biodegradation of $n$-alkanes $\mathrm{C}_{12}-\mathrm{C}_{30}$ and of various aromatic compound in the crude oil was (examined by GC/FID) using Acinetobacter. sp T4 culture. The n-alkanes were almost completely degraded, while the aromatic compounds were not. In the culture of Pseudomonas putida PB4, neither the nalkanes nor the aromatic compounds were degraded to any significant degree. In the mixed culture of Pseudomonas putida PB4 and Acinetobacter. sp. T4, however, both the n-alkanes and aromatic compound were degraded. ${ }^{[12]}$ Reported that GC profiles after 7 and 18 days showed that biodegradation of hydrocarbons of the crude oil fractions were totally utilized more than that in the control solutions. Reported that the capillary gas chromatographic analysis of the degraded crud oil revealed that crude oil components of chain length $\mathrm{C}_{12}$ to $\mathrm{C}_{32}$ were extensively degraded by Serratia marcescens OCS-21 after 16 days of incubation while 
1



2



3

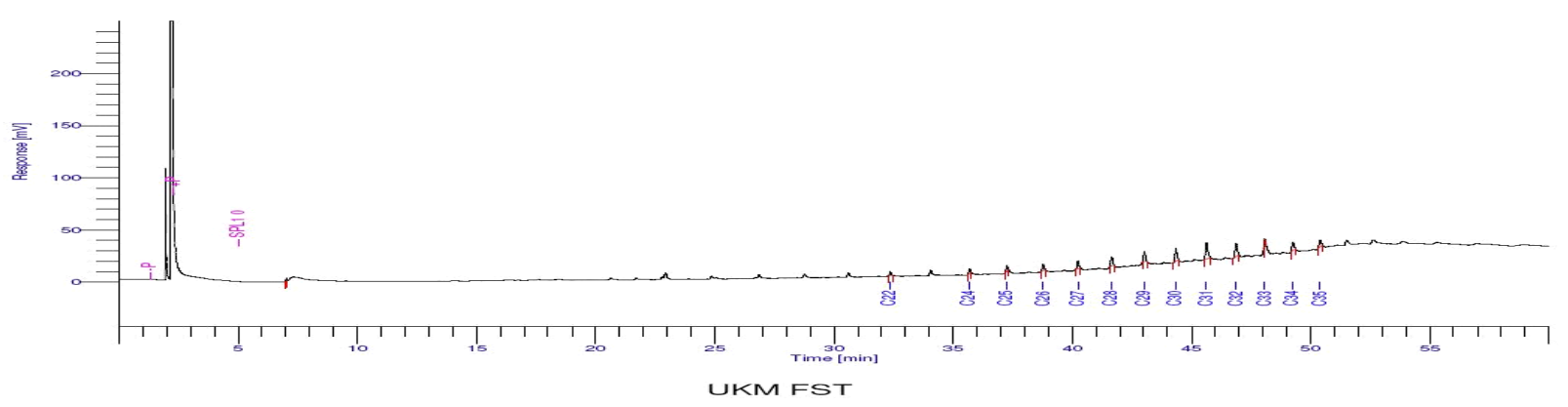

4

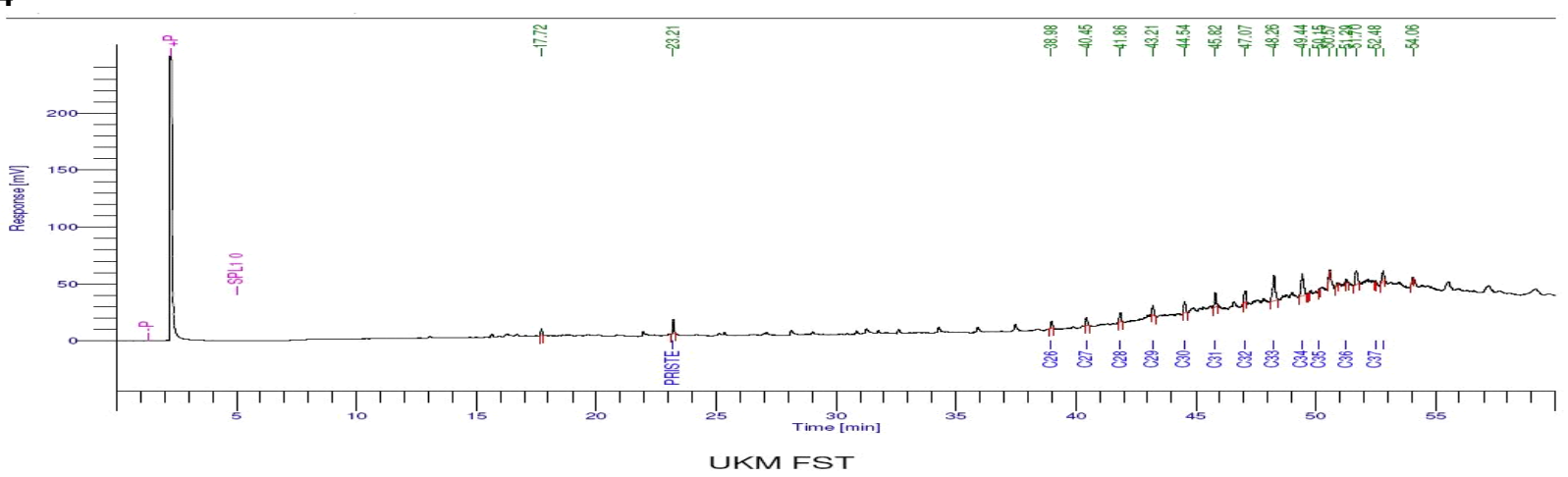

Fig. 4: Chromatogram of 1\% Tapis crude oil (1) before microbial degradation (2) degradation by microbial consortium $[\mathrm{AB}],(3)$ degradation by microbial consortium [AC], (4) degradation by microbial consortium [BC], after 5 days with MSM medium + peptone 
1



2

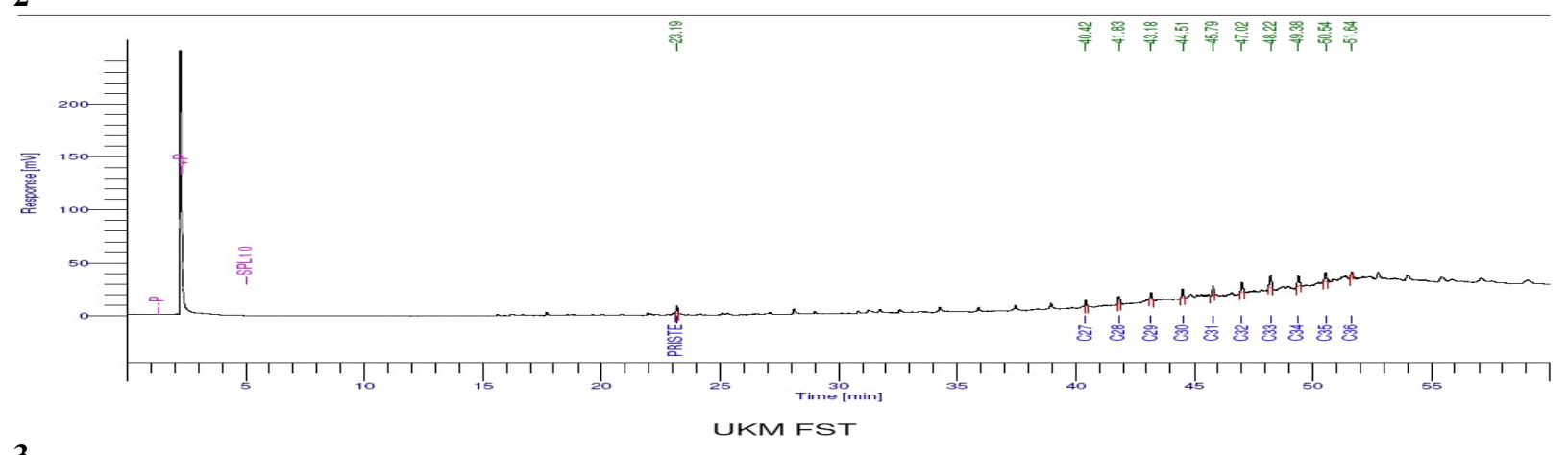

3

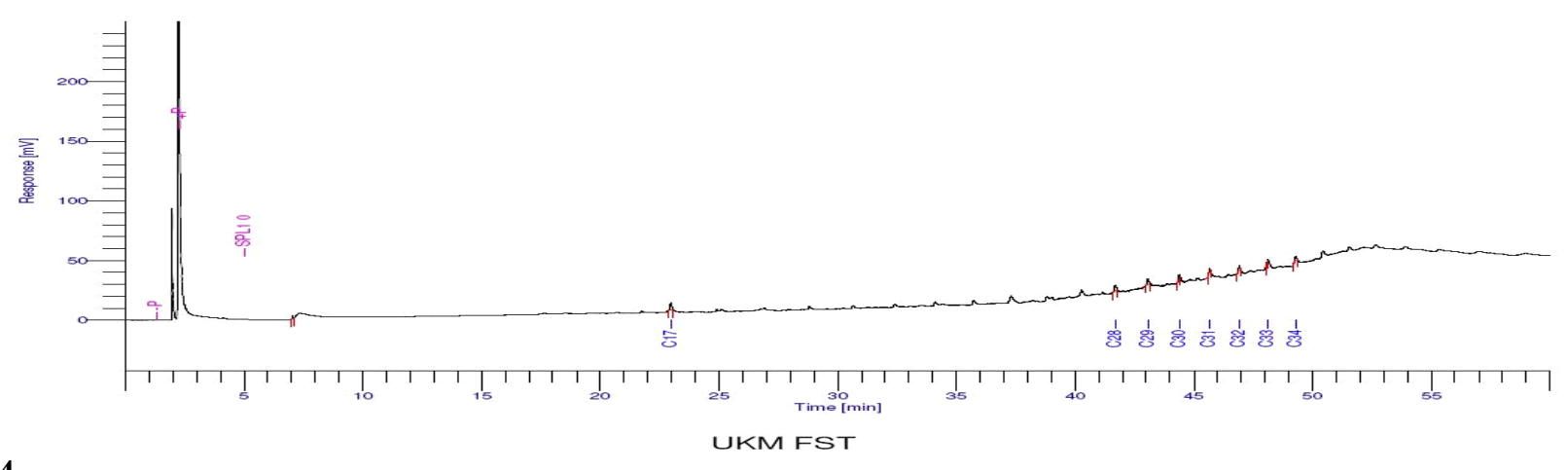

4

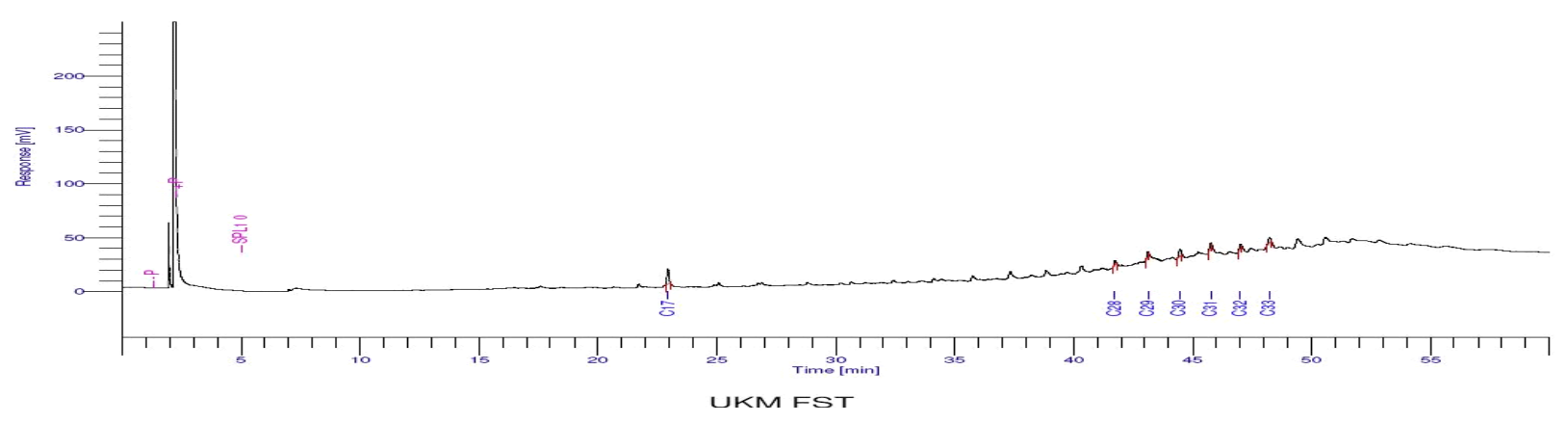

Fig. 5: Chromatogram of 1\% Tapis crude oil (1) before microbial degradation and degradation by microbial consortium [ABC], (2) after 5days, (3) after 10days and (4) after 15days with MSM medium + peptone 
Acinetobacter calcoaceticus COU-27 was able to degrade only $\mathrm{C}_{22}$ to $\mathrm{C}_{30}$ components of the crude oil after 16days of incubation. All four isolates showed a maximum of hydrocarbon removals which were seen with the short-chain and medium-chain aliphatic compounds compared to longer-chain aliphatic compounds. This is in agreement since short-chain and medium-chain alkanes are generally more easily degraded due to their lower hydrophobicity. This confirms our result that degradation by consortia is more effective than individual bacteria.

\section{CONCLUSION}

Two isolates (Acinetobacter faecalis WD2 and Staphylococcus. sp DD3) showed maximum growth in $1 \%$ peptone and two isolates (Neisseria elongate TDA4.2 and Pseudomonas putida TAM4.4) showed maximum growth in $0.5 \%$ peptone. Mixed bacterial consortium (ABC) gave a maximum of $98 \%$ degradation after 15 days incubation. With $100 \%$ of hydrocarbon removals were seen with the short-chain and medium chain alkanes compared to the longer chain alkanes.

\section{ACKNOWLEDGEMENT}

The authors would like to thank UKM and PETRONAS for the financial support provided to carry out this work.

\section{REFERENCES}

1. Lee, J.K., D. Park, B.U. Kim, J.I. Dong and S. Lee, 1998. Remediation of petroleum contaminated soil by fluidized thermal desorption. Waste Manage., 18: $\quad 503-507 . \quad$ DOI: $\quad 10.1016 /$ S0956053X(98)00135-4.

2. Collin, P.H., 2001. Dictionary of Ecology and the Environment. 4th Edn., Peter Collin Publishing, London

3. Alexander, M., 1999. Biodegradation and Bioremediation. 2nd Edn., Academic Press, San, Diego

4. Rambeloarisoa, E., J.F. Rontani, G. Giusti, Z. Duvnjak and J.C. Bertand, 1984. Degradation of crude oil by a mixed population of bacteria isolated from sea-surface foams. Mar. Biol., 83: 69-81. DOI: 10.1007/BF00393087.

5. Banat, I.M., R.S. Makkar and S.S. Cameotra, 2000. Potential commercial applications of microbial surfactants. Appl. Microbiol. Biotechnol., 53: 495508. DOI: $10.1007 / \mathrm{s} 002530051648$.
6. Zajic, E. and B. Supplisson, 1972. Emulsification and degradation of 'Bunker $\mathrm{C}$; fuel oil by microorganisms. Biotechnol. Bioeng., 14: 331343. http://www.ncbi.nlm.nih.gov/pubmed/5029879.

7. Chaillan, F., L.A. Fleche, E. Bury, Y-hui Phantavong, P. Grimont, A. Saliot and J. Oudot, 2004. Identification and biodegradation potential of tropical aerobic hydrocarbondegrading microorganisms. Res. Microbiol., 155: 587-595. DOI: 10.1016/j.resmic.2004.04.006.

8. Tano-Debrah, K., S. Fukuyama, N. Otonari, F. Taniguchi and M. Ogura, 1999. An noculum for the aerobic treatment of wastewaters with high concentration of fats and oils. Bioresour. Technol., 69:

133-139.

http://cat.inist.fr/?aModele $=$ afficheN\&cpsidt $=1743$ 226.

9. Zeng, F., K. Cui, X. Li, J. Fu and G. Sheng, 2004. Biodegradation kinetics of phthalateesters by Pseudomonas Fluoresences FS1. Proc. Biochem., 39: $\quad 1125-1129$. DOI: $10.1016 / \mathrm{S} 0032-$ 9592(03)00226-7.

10. Rahman, K.S.M., J. Thahira-Rahman, Y. Kourkoutas, I. Petsas, R. Marchant and I. M. Banat, 2003. Enhanced bioremediation of nalkane in petroleum sludge using bacterial consortium amended with rhamnolipid and micronutrients. Bioresour. Technol., 90: 159-168. DOI: 10.1016/S0960-8524(03)00114-7.

11. Komukai-Nakamura, S., K. Sugiura, Y. YamauchiInomata, H. Toki, k. Venkateswarana, S. Yamamoto, H. Tanaka and S. Harayama, 1996. Construction of bacterial consortia that degrade Arabian light crude oil. J. Fermentation Bioeng., 82: $\quad 570-574 . \quad$ DOI: $10.1016 /$ S0922338X(97)81254-8.

12. Nikolopoulou, M., N. Pasadakis and N. Kalogerakis, 2007. Enhanced bioremediation of crude oil utilizing lipophilic fertilizers. Desalination, 211: 286-295. DOI: 10.1016/j.desal.2006.02.095.

13. Ijah, U.J.J., 1998. Studies on relative capabilities of bacterial and yeast isolate tropical soil in degrading crude oil. Waste Manage., 18: 293-299. DOI: 10.1016/S0956-053X(98)00037-3. 